\title{
Observer-Based Direct Field Orientation: Analysis and Comparison of Alternative Methods
}

\author{
Patrick L. Jansen, Student Member, IEEE, Robert D. Lorenz, Senior Member, IEEE, and \\ Donald W. Novotny, Fellow, IEEE
}

\begin{abstract}
This paper focuses on methods of achieving direct field orientation (DFO) of induction machines based on closedloop, stator, and rotor flux observers which are well suited to both zero and very high-speed operation. Both observer topologies are dominated by a current model at zero and low speeds, and a voltage model at high speeds. Application of such rotor and stator flux observers to both stator and rotor direct field orientation is presented, including experimental results for three different methods.

The influence which flux regulation has on parameter sensitivity of the complete DFO system is analyzed. A rotor-flux-regulated and -oriented system is shown to be sensitive to leakage inductance under high slip (i.e., field weakened) operation. Both a stator-flux-regulated and -oriented system and a stator-fluxregulated, rotor-flux-oriented system are shown to have reduced parameter sensitivity at high speeds.

Unlike stator flux orientation using simple voltage integration stator flux models, excellent zero and low-speed operation of an observer-based stator-flux-oriented system is demonstrated.
\end{abstract}

\section{INTRODUCTION}

A CHIEVING high-quality torque and flux control in applications requiring both zero and very high-speed operation is difficult with existing approaches to induction machine field orientation. Indirect field orientation (IFO) utilizing a shaft encoder or resolver is the most common means, especially when zero-speed operation is required [1]. However, controller detuning can result in a substantial deterioration of performance. IFO is particularly parameter sensitive in large machines, high-efficiency machines, and when field weakening at high speeds [2].

Direct field orientation (DFO) based upon estimation of either the rotor or stator flux from the terminal voltage and current is an alternative approach that is very attractive for high-speed, field-weakened operation. At high speeds, the voltage model provides an accurate stator flux

Paper IPCSD 94-22, approved by the Industrial Drives Committee of the IEEE Industry Applications Society for presentation at the 1993 IAS Annual Meeting. Manuscript released for publication March 24, 1994. This work was supported by the Wisconsin Electric Machines and Power Electronics Consortium (WEMPEC) of the University of WisconsinMadison.

P. L. Jansen is with the Morrison Knudsen Corporation, Boise, ID 83705 .

R. D. Lorenz and D. W. Novotny are with the Department of Electrical and Computer Engineering, University of Wisconsin-Madison, Madison, WI 53706.

IEEE Log Number 9402641 estimate because the machine back EMF dominates the measured terminal voltage. However, at low speeds, the stator IR drop becomes significant, causing the accuracy of the flux estimate to be sensitive to the estimated stator resistance. Due to that sensitivity and to inherent signal integration problems at low excitation frequencies, DFO systems based solely upon the voltage model are generally not capable of achieving high dynamic performance at low and zero speeds [3]-[7].

A DFO approach was proposed in [8]-[10] that effectively combines the best accuracy attributes of IFO and voltage model DFO by utilizing a specific topology of closed-loop rotor flux observer. That topology of closedloop observer provides a smooth, deterministic transition between flux estimates produced by two different rotor flux models. A prior approach to provide a smooth transition between models was developed by Takahashi and Noguchi. They combined two stator flux models via a simple first-order lag-summing network [11]. In addition, rather than performing DFO, their stator flux estimate was used to select appropriate voltage vectors for direct torque control.

While [8] focused on the general design and accuracy analysis of both open- and closed-loop rotor flux observers, and [9] proposed the closed-loop rotor flux observer for DFO in wide speed range applications, this paper analyzes and compares different approaches to observer-based DFO. Both stator and rotor flux DFO systems based upon stator and rotor closed-loop flux observers are analytically and experimentally evaluated. Particular emphasis is placed on parameter sensitivity in the field-weakening region. Unlike in [10], measured rotor position is assumed to be available.

\section{Rotor Flux DFO SystemS}

The rotor and stator flux production in an induction machine can be modeled by the block diagram in Fig. 1, where the superscript " $e$ " denotes an arbitrarily aligned synchronous frame. Cross coupling in the form of a rotor flux-slip frequency product is clearly evident. (Note that this is the same cross coupling that is recognized as back EMF when modeled in the stationary frame.)

As is well known, orientation to the rotor flux (i.e., using rotor flux as the $d$-axis reference frame, $e \rightarrow r f$ ) 


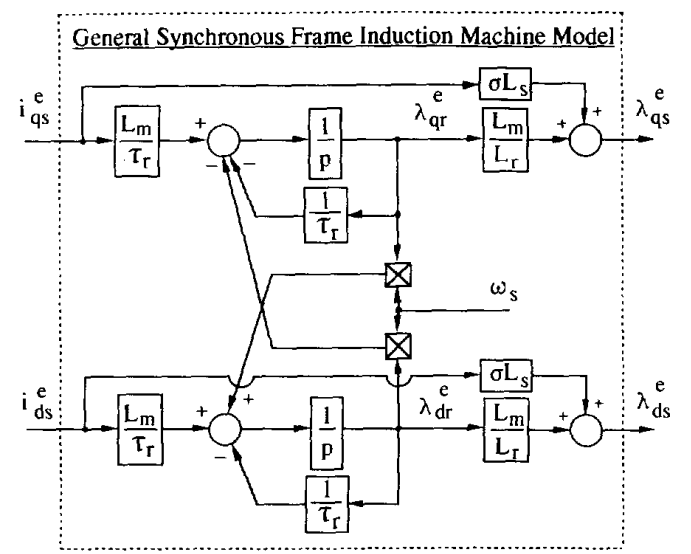

Fig. 1. Current input, flux output model of the induction machine in an arbitrarily aligned synchronous frame.

Such that $\lambda_{q r}^{r f}=0$, eliminates the cross coupling between $i_{q s}^{r f}$ and $\lambda_{d r}^{r f}$. Note, however, that $\lambda_{q s}^{r f} \neq 0$. With good current regulation, a correctly tuned rotor-flux-oriented system as shown in Fig. 2 exhibits decoupled control of torque and rotor flux.

Rather than directly calculating the $d$-axis current command in a feedforward approach, as is common in indirect field orientation (IFO), a flux regulator $G_{f c}$ is typically used in DFO systems. One purpose of the regulator is to compensate for potential phase lags in the current regulator. Current phase lags result in increased $d$-axis current which attempts to build the flux beyond the commanded level. The flux regulator counteracts by decreasing the $d$-axis current command, effectively advancing the current angle. As will be shown, the flux regulator can also reduce parameter sensitivity.

By calculating the torque current command $i_{q s}^{r f^{*}}$, using the estimated rotor flux (feedback) rather than the commanded rotor flux, dynamic torque control is virtually independent of the flux regulator bandwidth.

Unfortunately, the rotor flux is not directly measurable, and thus significant errors can exist in the estimated rotor flux feedback signal, and hence also in the actual operating flux and the actual developed torque.

\section{A Closed-LoOp Rotor Flux ObSERVER}

The closed-loop rotor flux observer proposed in [8]-[10] is illustrated in Fig. 3 in block diagram form using complex vector variables. Unlike indirect field orientation where flux is estimated in the synchronous frame, this approach is implemented primarily in the stationary frame. The closed-loop observer is formed from two open-loop rotor flux observers which are referred to as the current and voltage models. The current model utilizes measured stator current and rotor position to produce a flux estimate, while the voltage model relies on the measured stator voltage and current. The smooth transition between current and voltage model flux estimates is governed by the closed-loop eigenvalues (observer bandwidth) deter-

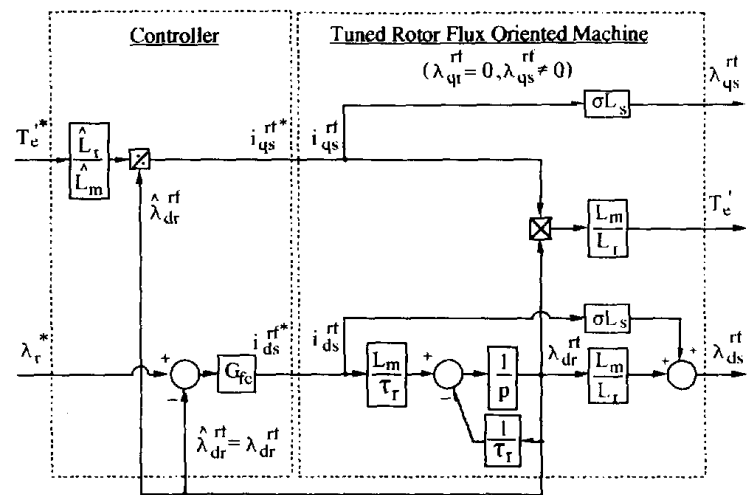

Fig. 2. A tuned rotor-flux-regulated rotor-flux-oriented (RFR-RFO) system assuming ideal current regulation. ( $G_{f c}$ is a flux regulator, e.g., a simple PI controller.)

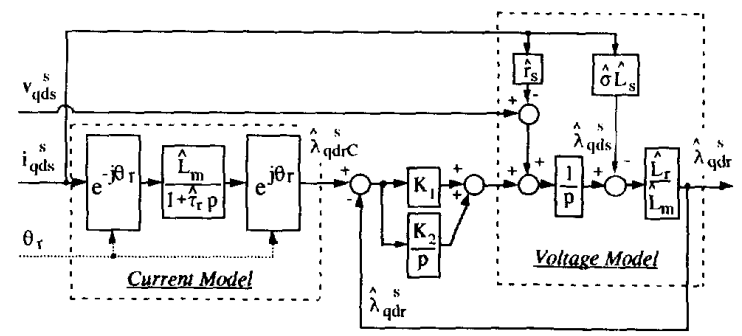

Fig. 3. Closed-loop rotor flux observer based upon the current and voltage rotor flux models in complex vector notation.

ministically set by gains $K_{1}$ and $K_{2}$ of the linear controller.

Note that the current model is best implemented in the rotor frame (i.e., physical rotor, not rotor flux frame) and thus, as depicted in Fig. 3, requires transformations between the stationary and the rotor frames using the measured rotor position. When implemented in the stationary frame, the current model requires measured rotor velocity and has velocity-dependent cross coupling that can lead to numerical instabilities at higher velocities when digitally implemented. (Note that this is the same cross coupling present in Fig. 1.) Transformation to the rotor frame completely eliminates the undesirable cross coupling, and permits the use of rotor position which, unlike velocity, is generally directly available and sufficiently accurate.

Under field-oriented operation, the slip is generally small, and rotor velocity corresponds closely to excitation frequency. This causes the observer estimate to transition between the current and voltage models very nearly in proportion to the rotor speed. The flux estimation frequency response function (FRF) from [9] and replotted in Fig. 4 relates the estimated flux to the actual rotor flux for rated slip operation using gains set to achieve $10 \mathrm{~Hz}$ closed-loop eigenvalues. The flux observer sensitivity to parameter estimates corresponds to that of the current model at velocities below the observer bandwidth, and to the voltage model at velocities above the bandwidth. 

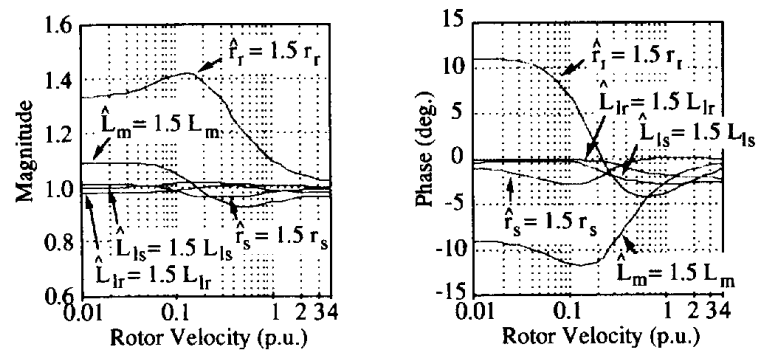

Fig. 4. Closed-loop rotor flux estimation FRF, $\hat{\lambda}_{q d r} / \lambda_{q d r}$, illustrating parameter sensitivity of the closed-loop rotor flux observer at rated slip operation ( $10 \mathrm{~Hz}$ eigenvalues (0.17 p.u.), (A3) in Appendix).

\section{Flux Regulation And Current Model Parameter Sensitivity}

The current model was shown in [9] to be the same model used for indirect field orientation (IFO). A DFO system based on the current model would thus be expected to have the same sensitivity to the rotor time constant and the magnetizing inductance. However, from the current model flux estimation FRF phase plot in Fig. 5 , one might incorrectly conclude that the parameter sensitivity of a flux-regulated DFO system would be reduced at high operating slip frequencies $\omega_{s}$ (corresponding to the field-weakened operation). Despite the flux estimation phase accuracy improvement, errors in the rotor time constant will now be shown to cause errors via an incorrect operating slip, similar to IFO slip frequency calculation error.

Fig. 6(a) shows the slip frequency to which the DFO system will converge, as a function of the effective DFO commanded slip frequency $\omega^{*}$, i.e.,

$$
\omega_{s}^{*}=\frac{L_{m}}{\tau_{r}} \frac{i_{q s}^{r f *}}{\lambda_{r}^{*}},
$$

for errors in rotor resistance estimation. As expected, the operating slip frequency is the same as the commanded slip frequency of an IFO system. The corresponding operating flux level is plotted in Fig. 6(b). Also plotted in Fig. 6(b) is the estimated flux level, which for rotor resistance errors agrees with the commanded level, i.e, incorrect orientation due to flux estimate phase errors results in flux level errors equivalent to the estimation error. In other words, both flux phase and magnitude errors result in equivalent operating errors in the DFO system.

Given this inherent property, the flux regulator has no influence with respect to rotor resistance errors.

Fig. 7(a) and (b) plot the corresponding operating slip frequency and flux level for errors in magnetizing inductance estimation. Unlike the case with rotor resistance errors, the estimated flux level [also plotted in Fig. 7(b)] is not in agreement with the commanded level, i.e., flux phase and magnitude errors do not result in equivalent operating errors in the DFO system.

However, the DFO flux regulator will drive the estimated magnitude to the commanded level. The resulting
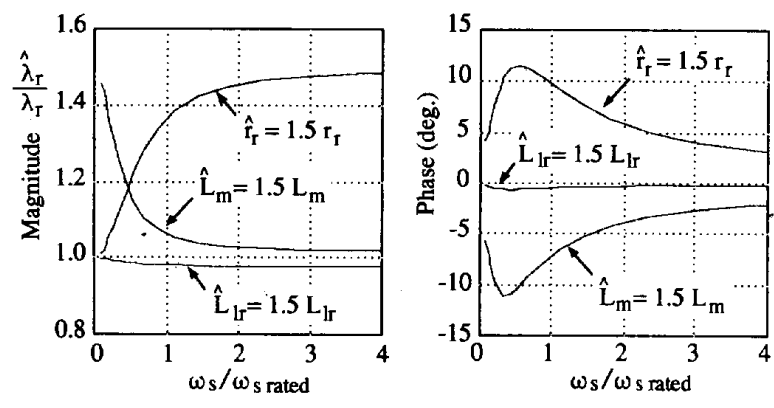

Fig. 5. Current model FRF, $\hat{\lambda}_{q d r c}^{s} / \lambda_{q d r}^{s}$, illustrating the parameter sensitivity as a function of operating slip frequency [(A1) in Appendix)].
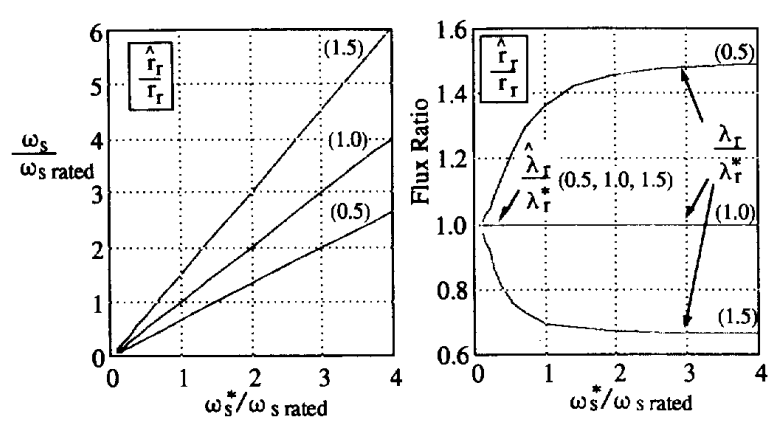

Fig. 6. Resulting (a) slip frequency and (b) flux of a DFO system based upon the current model as a function of effective commanded slip frequency for rotor resistance parameter errors.
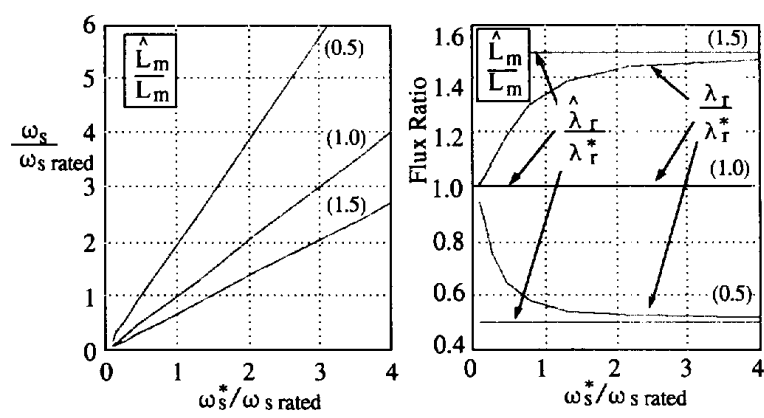

Fig. 7. Resulting (a) slip frequency and (b) flux of a DFO system based upon the current model as a function of effective commanded slip frequency for magnetizing inductance parameter errors.

operating point will then correspond to the level indicated by the FRF magnitude plot in Fig. 5 .

If the flux feedback regulator is replaced with the feedforward approach used in IFO such that

$$
i_{d s}^{*}=\frac{1}{\hat{L}_{m}}\left[1+\hat{\tau}_{r} p\right] \lambda_{r}^{*}
$$

the DFO system will converge on the same operating point as IFO.

Errors in the estimated rotor leakage inductance influence the system in the same manner as the rotor resistance, although at a greatly reduced level. Thus, a major 
conclusion, although not surprising, is that under steadystate conditions, the flux regulator offers no advantage over the feedforward approach used in IFO systems with respect to parameter sensitivity of the current model in DFO systems. The flux regulator will be shown, however, to significantly improve the DFO system when using the voltage model.

\section{Parameter Sensittvity in the Field-Weakening Region}

At high speeds, especially in the field-weakening/constant-horsepower region, both the motor and the drive tend to be highly stressed. It is under these operating conditions that the system is most sensitive to losses and voltage limits, and hence to accurate field orientation. The accuracy of the flux estimate used for DFO which originates from the voltage model at these high speeds is thus of great importance.

Although the closed-loop rotor flux observer FRF plot in Fig. 4 indicates that the rotor flux estimate is only mildly sensitive to the leakage inductance estimates at rated slip frequency, the sensitivity increases with operating slip frequency. Fig. 8 plots the voltage model FRF as a function of slip frequency with varying errors in the rotor leakage inductance.

At high slip frequencies common to field-weakened operation, the magnitude and phase errors are substantial. With closed rotor slots, the rotor leakage inductance can easily vary by a factor of 2 or more. Because the stator leakage variation is much smaller due to the open stator slots, the corresponding stator leakage plots are not included.

The sensitivity to magnetizing inductance estimate is insignificant relative to the leakage inductance sensitivity.

Without flux regulation (i.e., via a feedforward approach), the phase errors in Fig. 8 will cause the DFO system to converge on an incorrect operating slip and hence flux level, as plotted in Fig. 9. Note that the actual flux errors are significantly greater than the flux estimate magnitude errors in Fig. 8.

With rotor flux regulation, the DFO system will converge to a more correct flux level indicated by the FRF magnitude plots in Fig. 8. Although a thorough stability analysis is beyond the scope of this paper, system stability can be shown to be greatly improved by the addition of the flux regulator.

Even with flux regulation, however, the potential error in the operating flux level due to errors in the rotor leakage inductance estimate can limit and/or significantly degrade very high-speed operation. Because stator flux is obtained directly from the stator voltage, it is not susceptible to leakage inductance parameter errors, and thus orientation to the stator flux has attracted considerable attention.

\section{A Closed-Loop Stator Flux ObServer}

A closed-loop stator flux observer structured similar to the closed-loop rotor flux observer is shown in Fig. 10.
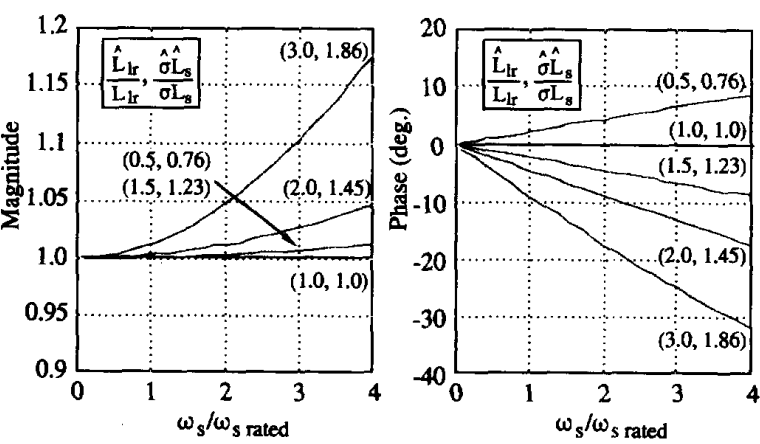

Fig. 8. Open-loop voltage-model rotor flux estimation FRF, $\hat{\lambda}_{q d r} / \lambda_{q d r}$, illustrating dependence of rotor leakage sensitivity on the operating slip. The corresponding error in the stator transient inductance is also indicated [(A2) in Appendix].
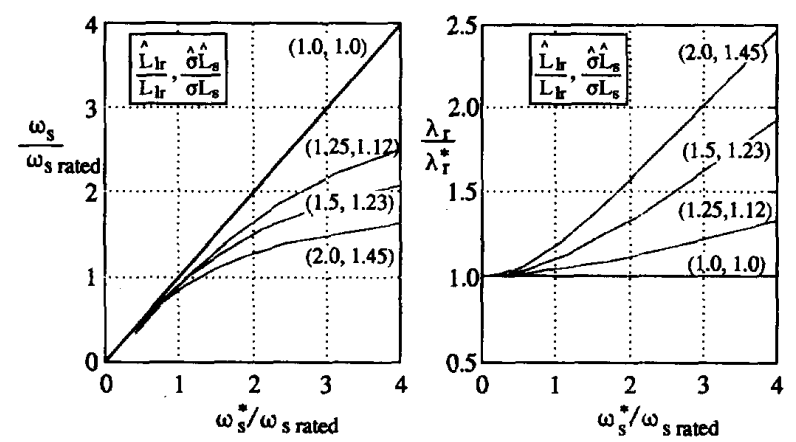

Fig. 9. Resulting (a) slip and (b) rotor flux of a DFO system without flux regulation based upon the voltage model as a function of effective commanded slip (1) for rotor leakage inductance parameter errors.

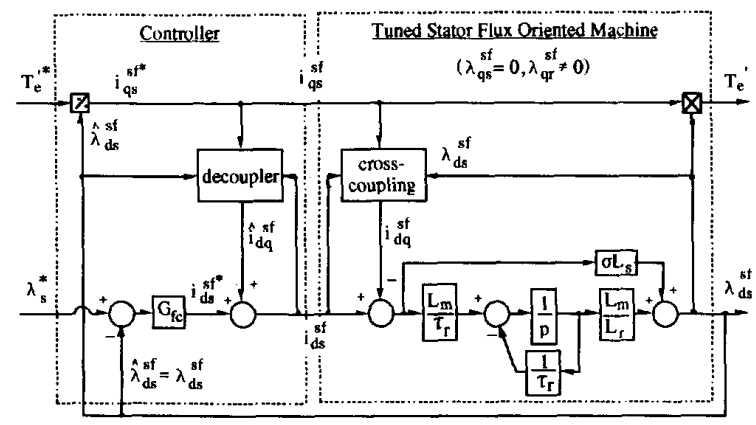

Fig. 10. A closed-loop stator flux observer of identical topology to the rotor flux observer in Fig. 3.

Note that the leakage terms have simply moved from the rotor flux voltage model to the stator flux current model, and thus the net computation and parameters required by the closed-loop observer are unchanged.

The FRF relating the estimated to the actual stator flux for rated slip operation is plotted in Fig. 11 with gains set for $10 \mathrm{~Hz}$ closed-loop eigenvalues. The parameter sensitivity is nearly identical to that of the rotor flux observer, the major difference being the sensitivity to leakage induc- 

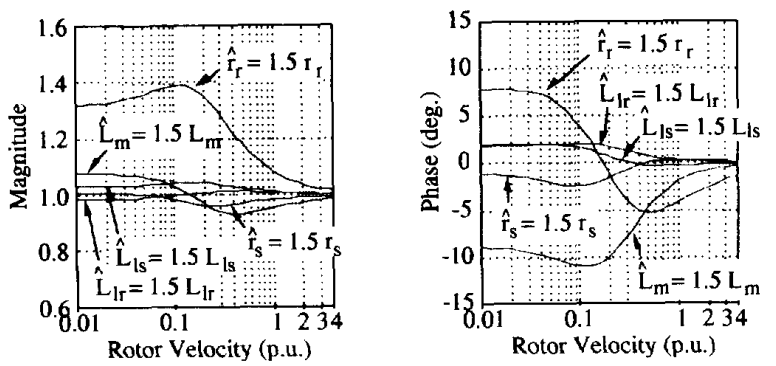

Fig. 11. Closed-loop FRF, $\hat{\lambda}_{q d s} / \lambda_{q d s}$, illustrating parameter sensitivity of the closed-loop stator flux observer at rated slip operation $(10 \mathrm{~Hz}$ eigenvalues) [(A6) in Appendix].

tance which has moved from the voltage model to the current model. For less than rated slip frequency, characteristic of low-speed operation below rated torque, the increased leakage sensitivity of the current model is small relative to the rotor resistance and magnetizing inductance sensitivities. The observer also functions at zero speed.

\section{A Stator Flux DFO System}

In addition to the elimination of parameter sensitivity at high speeds, orientation and regulation of the stator flux can result in nearly optimal torque capability over the entire field-weakening speed range [8].

A significant difference between stator and rotor flux orientation is the level of cross coupling that occurs between the $d$ and $q$ axes. When oriented to the rotor flux (i.e., $\lambda_{q r}^{r f}=0$ ), cross coupling of the stator $q$-axis current to the $d$-axis rotor flux is completely eliminated, as was shown in Fig. 2. However, with stator flux orientation (i.e., $\lambda_{q s}^{s f}=0$ ), the $q$-axis rotor flux is not zero, and thus a significant amount of cross coupling still exists. This cross coupling can be expressed in the form of a crosscoupling current $i_{d q}^{\text {sf }}$ acting as a flux producing input in parallel with $i_{d s}^{s f}$ as shown in Fig. 12.

For dynamic flux control, a decoupler, also shown in Fig. 12, can be implemented to eliminate the undesirable cross coupling [3]. Unfortunately, the decoupler is parameter sensitive and requires additional computation.

A stator-flux-regulated stator-flux-oriented (SFR-SFO) DFO system complete with decoupler is shown in Fig. 13. Since the torque command ( $q$-axis) current is calculated via the estimated stator flux which is most accurate at high speeds (assuming negligible voltage measurement and integration errors), the dynamics and accuracy of the developed torque are dependent solely upon the current regulation. The flux regulator drives the stator flux to the desired level. However, the decoupler can be removed, causing dynamic stator flux variations dependent on flux regulator bandwidth.

\section{A Rotor-FluX-Oriented Stator Flux REGULATED DFO SYSTEM}

The attractive features of the SFR-SFO system in Fig. 13 are not unique to stator flux orientation, but rather to

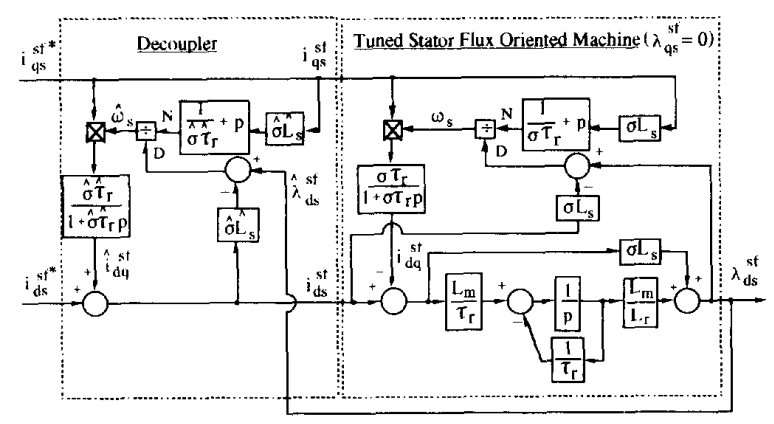

Fig. 12. A tuned stator-flux-oriented machine model illustrating cross coupling and a control-based decoupler.

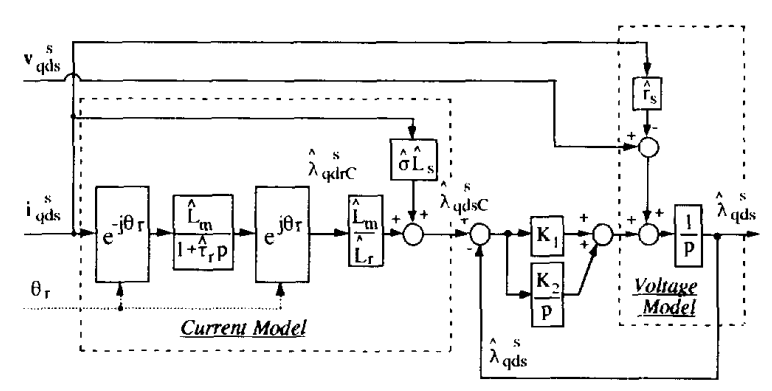

Fig. 13. A stator-flux-regulated stator-flux-oriented (SFR-SFO) DFO system with decoupler $\left(G_{f c}=\right.$ stator flux regulator, e.g., PI controller).

stator flux regulation. The stator-flux-regulated rotorflux-oriented (SFR-RFO) system illustrated in Fig. 14 is also capable of achieving correct torque and flux control, even under detuned conditions.

Compared to the previous rotor and stator-flux-oriented systems, the torque command current must now be calculated via the full torque equation in the rotor flux frame, i.e.,

$$
i_{q s}^{r r^{*}}=\left(T_{e}^{* *}+\hat{\lambda}_{q s}^{r f} i_{d s}^{r f^{*}}\right) / \hat{\lambda}_{d s}^{r f} .
$$

In addition, the stator flux magnitude must also be calculated, although the computationally expensive square root function can be avoided by simply regulating the square of the stator flux magnitude.

To gain perspective on the different possible DFO systems, a rotor-flux-oriented machine detuned with respect to the stator transient inductance is illustrated in Fig. 15. The model is derived in [7]. The cross coupling due to detuning is of the same form as that always present in the stator-flux-oriented system in Fig. 12.

The control-based decoupler in Fig. 12 attempts to remove the cross coupling that is not present in the tuned rotor-flux-oriented system. Because the decoupler is sensitive to additional machine parameters such as the rotor time constant, correct decoupling in the stator-flux-oriented system is more difficult than in the rotor-flux-oriented system.

The rotor flux orientation attempts to maintain constant rotor flux under torque command changes, while 


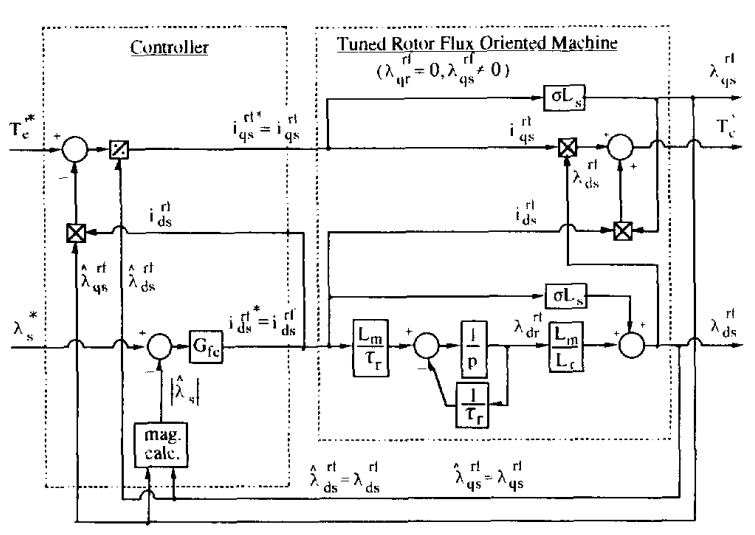

Fig. 14. A tuned, stator-flux-regulated rotor-flux-oriented (SFR-RFO) DFO system.

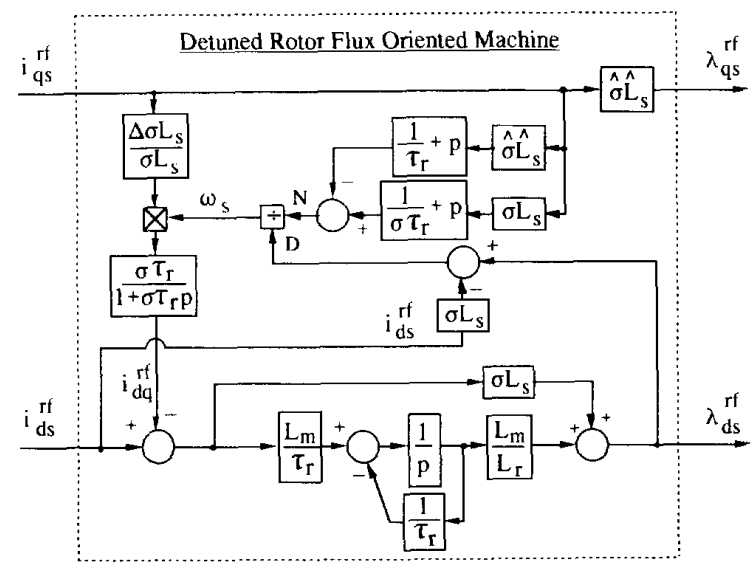

Fig. 15. A rotor-flux-oriented machine with detuned stator transient inductance $\left(\Delta \sigma L_{s}=\sigma L_{s}-\hat{\sigma} \hat{L}_{s}\right)$.

allowing the stator flux to change, i.e., $\lambda_{q r}^{r f}=0$, but $\lambda_{q s}^{r f}=$ $\hat{\sigma} \hat{L}_{s} r_{q s}^{r f} \neq 0$. The stator flux regulator counteracts by attempting to maintain constant stator flux. Thus, a tuned rotor-flux-oriented stator-flux-regulated system with limited flux regulation bandwidth will experience a perturbation in the stator flux magnitude under torque command changes. The perturbation is not present in the statorflux-oriented system with a correct decoupler, but is present at a potentially larger degree when either detuned or the decoupler is eliminated.

\section{Implementation of Observer Based Direct FiELD ORIENTATION}

The three observer-based DFO systems designated RFR-RFO, SFR-RFO, and SFR-SFO were experimentally evaluated via the implementations in Fig. 16. The observers and coordinate transformations were implemented in software on the Motorola DSP56001 with a 4 $\mathrm{kHz}$ sample rate. A faster timed interrupt routine sampled and averaged the measured voltages and currents (after the anti-alias filters), providing an effective integra-

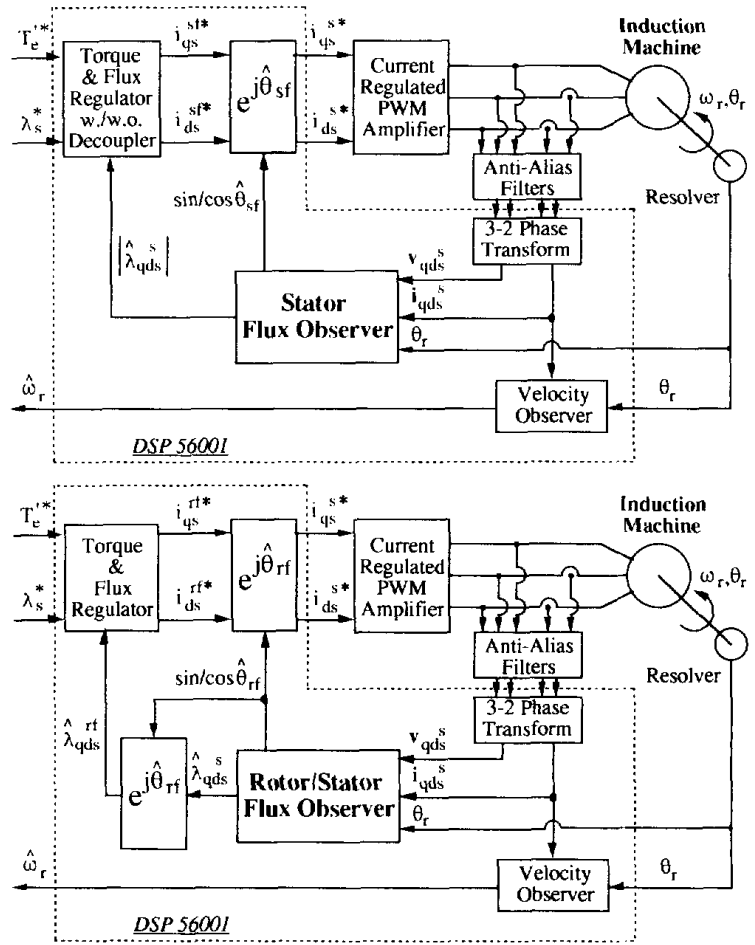

Fig. 16. Experimental DFO systems using closed-loop flux observers. Upper diagram: stator-flux-regulated stator-flux-oriented (SFR-SFO). Lower diagram: stator-flux-regulated rotor-flux-oriented (SFR-RFO). The rotor-flux-regulated rotor-flux-oriented (RFR-RFO) system implementation was of the same form as the SFR-SFO system without the decoupler. All three were implemented with a Motorola DSP56001.

tion step of $15.6 \mu \mathrm{s}(64 \mathrm{kHz})$. The PWM VSI switched at $3.4 \mathrm{kHz}$ with a stationary frame PI current regulator with $\approx 350 \mathrm{~Hz}$ bandwidth. Due to signal noise, the flux regulator bandwidth was limited to real eigenvalues of $\approx 15-20$ Hz. The flux observer two eigenvalues were set at 0.5 and $5.0 \mathrm{~Hz}$.

Experimental results illustrating the response of the three systems to square-wave torque commands toggled between velocity limits are shown in Figs. 17-20. The load was dominated by the rotor inertia with negligible damping; thus, a triangular velocity waveform would be expected for a tuned system.

Under rated conditions and with a reasonably high flux regulator bandwidth, all three systems behaved similarly, providing good torque and flux control, independent of detuning and/or the SFO decoupler.

Zero- and low-speed operation of the SFR-SFO system is demonstrated in Fig. 17 under tuned and detuned $\left(\hat{r}_{r}=2.0 r_{r}\right)$ conditions with a rated torque command. As expected (see Fig. 11), some sensitivity to $\hat{r}_{r}$ is present from the stator flux current model.

To demonstrate the differences among the three DFO approaches, the systems were pushed to the limits, i.e., wide-speed operation up to \pm 2.7 p.u., field weakening to $1 / 3$ rated flux, $1 / 3$ rated torque commands, and severe detuning to $\hat{L}_{l r}=3.0 L_{l r}$ (see Figs. 18-20). The resulting 

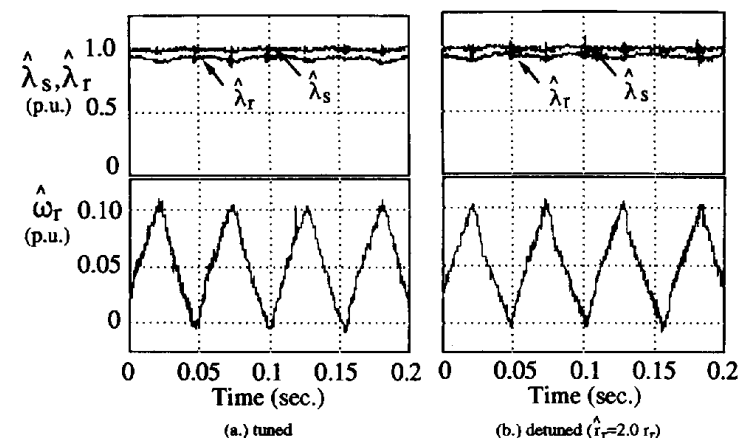

Fig. 17. Experimental low-speed results for estimated rotor and stator flux magnitudes and rotor velocity for the SFR-SFO system without decoupler under tuned and detuned conditions.

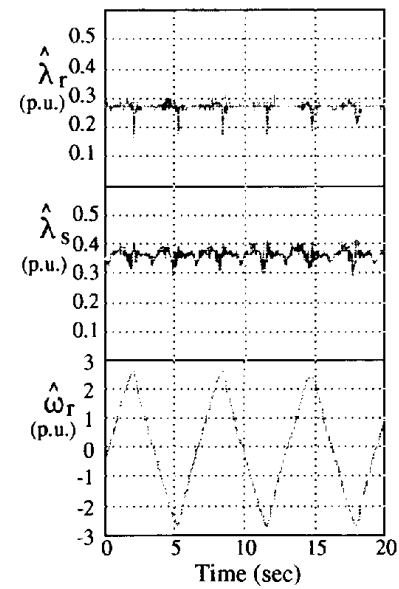

(a.) tuned

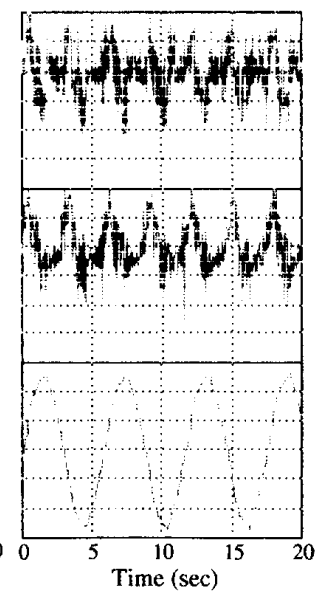

(b.) detuned $\left(\hat{L}_{\mathrm{tr}}=3.0 \mathrm{~L}_{\mathrm{Lr}}\right)$

Fig. 18. Experimental results for estimated rotor and stator flux and rotor velocity for the RFR-RFO system under tuned and detuned conditions.
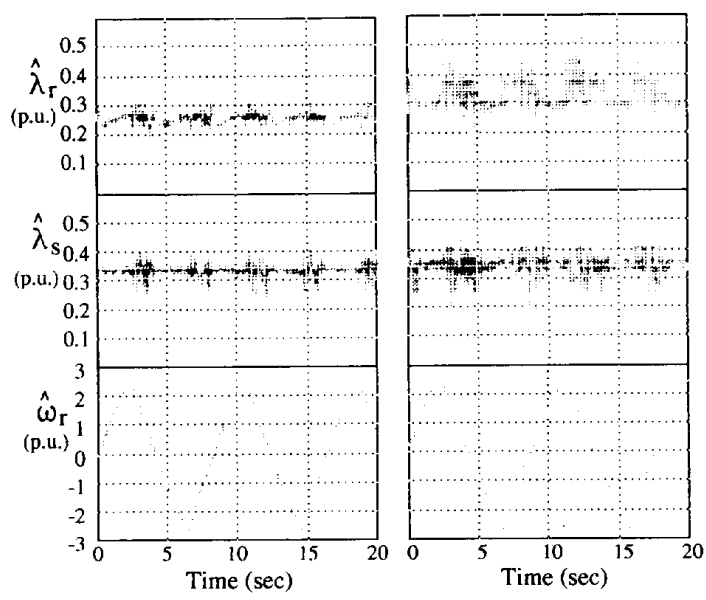

(a.) tuned

(h.) detuned $\left(\hat{(}_{t_{r}}=3.0 L_{t r}\right)$

Fig. 19. Experimental results for estimated rotor and stator flux and rotor velocity for the SFR-RFO system under tuned and detuned conditions.

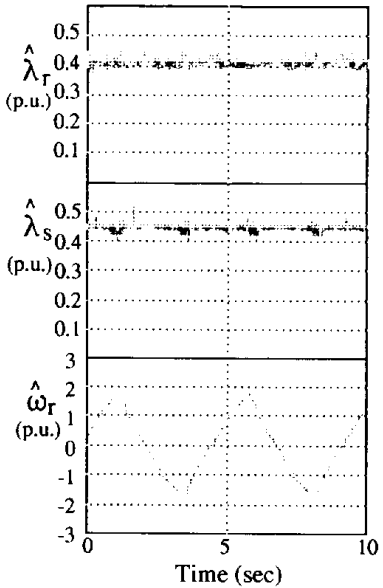

(a.) tuned decoupler

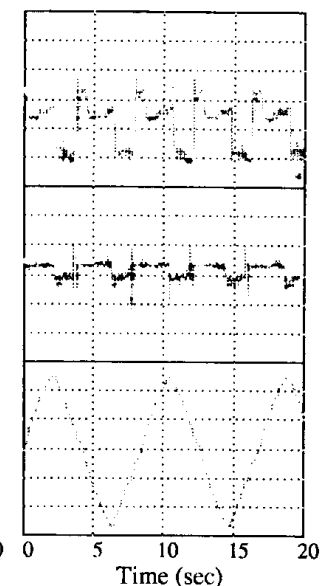

(b.) without decoupler

Fig. 20. Experimental results illustrating estimated rotor and stator flux magnitudes and rotor velocity for the SFR-SFO system with (a) tuned decoupler and (b) without decoupler.

high-slip operation was shown to increase the sensitivity to the leakage estimates. The flux regulator is forced to compensate for errors attributable to poor parameter estimates, the chosen orientation reference, and to the stationary frame PI current regulator limits.

In Fig. 18, the RFR-RFO system performed well when tuned and field weakened, but not detuned and field weakened. In Fig. 19, the SFR-RFO system performed nearly as well as the RFR-FRO system when tuned and field weakened, but was substantially better than the RFR-RFO in the detuned, field-weakened operation. In Fig. 20, the SFR-SFO system with the tuned decoupler performed excellently up to intermediate speeds $\approx 1.7$ p.u. velocity. At speeds greater than this, the decoupled system gave evidence of instabilities. In Fig. 20, the SFR-SFO system without the decoupler operated adequately at higher speeds, although the cross coupling was substantially more than the flux regulator could easily compensate for.

\section{$\mathrm{X}$. Conclusions}

This paper presented an analysis and comparison of different methods of achieving observer-based direct field orientation (DFO). DFO based upon rotor and stator flux closed-loop observers is an excellent means of achieving flux and torque control over very wide speed ranges including zero and field-weakened regions. The approach has substantially improved parameter insensitivity compared to existing methods.

Three observer-based DFO approaches were evaluated.

- Below rated operation, all showed good performance.

- Zero- and low-speed operation of stator-flux-observer, stator-flux-oriented control was demonstrated.

The three DFO approaches differed when the system limits were pushed. 
- The rotor-flux-regulated and -oriented system was shown to be highly sensitive to leakage inductance estimates under high-slip operation.

- A stator-flux-regulated and -oriented system without decoupler is parameter insensitive at high speeds, but suffers from excessive cross coupling under high-slip operation that must be handled by the flux regulator.

- A stator-flux-regulated and rotor flux-oriented system appears to offer intermediate parameter sensitivity and cross coupling which is substantially easier to remove than with stator flux regulation and orientation.

\section{APPENDIX}

A. Current Model, Voltage Model, and Closed-Loop Observer FRF Equations

Rotor Flux Observers:

$$
\begin{gathered}
\frac{\hat{\lambda}_{q d r C}^{s}}{\lambda_{q d r}^{s}}=\frac{\hat{L}_{m}}{L_{m}}\left(\frac{1+j \tau_{r} \omega_{s}}{1+j \hat{\tau}_{r} \omega_{s}}\right)=\mathrm{FRF}_{R C} \\
\frac{\hat{\lambda}_{q d r}^{s}}{\lambda_{q d r}^{s}}=\frac{L_{m}}{L_{r}} \frac{\hat{L}_{r}}{\hat{L}_{m}}\left[1+\frac{L_{r}}{L_{m} 2}\left(1+j \tau_{r} \omega_{s}\right)\right. \\
\left.\cdot\left(\left(\sigma L_{s}-\hat{\sigma} \hat{L}_{s}\right)-j \frac{r_{s}-\hat{r}_{s}}{\omega_{e}}\right)\right]=\mathrm{FRF}_{R V} \\
\frac{\hat{\lambda}_{q d r}^{s}}{\lambda_{q d r}^{s}}=\frac{\hat{L}_{r}}{\hat{L}_{m}}\left(\mathrm{FRF} F_{R C}\right)+j \omega_{e}\left(\mathrm{FRF} F_{R V}\right) \\
K \frac{\hat{L}_{r}}{\hat{L}_{m}}+j \omega_{e} \\
\text { where } \boldsymbol{K}=K_{1}+K_{2} / p
\end{gathered}
$$

Stator Flux Observers:

$$
\begin{gathered}
\frac{\hat{\lambda}_{q d s C}^{s}}{\lambda_{q d s}^{s}}=\frac{\hat{L}_{s}}{L_{s}}\left(\frac{1+j \tau_{r} \omega_{s}}{1+j \hat{\tau}_{r} \omega_{s}}\right)\left(\frac{1+j \hat{\sigma} \hat{\tau}_{r} \omega_{s}}{1+j \sigma \tau_{r} \omega_{s}}\right)=\mathrm{FRF}_{S C} \\
\frac{\hat{\lambda}_{q d s}^{s}}{\lambda_{q d s}^{s}}=1-j \frac{1}{L_{s}}\left(\frac{1+j \tau_{r} \omega_{s}}{1+j \sigma \tau_{r} \omega_{s}}\right)\left(\frac{r_{s}-\hat{r}_{s}}{\omega_{e}}\right)=\mathrm{FRF}_{S V} \\
\frac{\hat{\lambda}_{q d s}^{s}}{\lambda_{q d s}^{s}}=\frac{\boldsymbol{K}\left(\mathrm{FRF}_{S C}\right)+j \omega_{e}\left(\mathrm{FRF}_{S V}\right)}{\boldsymbol{K}+j \omega_{e}}
\end{gathered}
$$

\section{B. Induction Machine Parameters}

Westinghouse TEE II, Frame $215 \mathrm{~T}, 10 \mathrm{hp}$, three phase, $460 / 230 \mathrm{~V}, 12.2 / 24.4 \mathrm{~A}, 1750 \mathrm{rpm}$, rated $\omega_{s} \approx 0.037$ p.u.

$$
\begin{array}{ll}
r_{s} \approx 0.20 \Omega & L_{l s} \approx 1.5 \mathrm{mH} \quad L_{m} \approx 32.3 \mathrm{mH} . \\
r_{r}=0.20 \Omega & L_{l r} \approx 1.5 \mathrm{mH}
\end{array}
$$

\section{ACKNOWLEDGMENT}

The authors wish to acknowledge the motivation provided by the Wisconsin Electric Machines and Power Electronics Consortium (WEMPEC) of the University of Wisconsin-Madison.

\section{REFERENCES}

[1] D. W. Novotny and R. D. Lorenz, Eds., Introduction to Field Orientation and High Performance AC Drives, tutorial book from 1985 and 1986 IEEE-IAS Ann. Meet.

[2] K. B. Nordin, D. W. Novotny, and D. S. Zinger, "The influence of motor parameter deviations in feedforward field orientation drive system," IEEE Trans. Ind. Appl., vol. IA-21, pp. 1009-1015, July/ Aug. 1985.

[3] X. Xu, R. De Doncker, and D. W. Novotny, "A stator flux oriented induction machine drive," in Proc. 1988 Power Electron. Specialist's Conf., Kyoto, Japan, Apr. 1988. pp. 870-876.

[4] - "Stator flux orientation control of induction machines in the field weakening region," in Proc. IEEE-LAS Annu. Meet., Oct. 1988, pp. 437-443.

[5] X. Xu and D. W. Novotny, "Implementation of direct stator flux orientation control on a versatile DSP based system," in Proc. IEEE-LAS Annu. Meet., Oct. 1990.

[6] - - "Selecting the flux reference for induction machine drives in the field weakening region," in Proc. IEEE-LAS Annu. Meet., Oct. 1991.

[7] X. Xu, "Stator flux orientation--A robust control technique for induction machines," Ph.D. dissertation, Univ. Wisconsin, Madison, 1990.

[8] P. L. Jansen and R. D. Lorenz, "A physically insightful approach to the design and accuracy assessment of flux observers for field oriented induction machine drives," in Proc. IEEE-LAS Annu. Meet., Oct. 1992, pp. 570-577.

[9] P. L. Jansen, C. O. Thompson, and R. D. Lorenz, "Observer-based direct field orientation for both zero and very high speed operation," in Proc. PCC, Yokohama, Japan, Apr. 1993, pp. 432-437.

[10] P. L. Jansen and R. D. Lorenz, "Accuracy limitations of velocity and flux estimation in direct field oriented induction machines," in Proc. EPE Conf., Brighton, England, Sept. 1993.

[11] I. Takahashi and T. Noguchi, "A new quick-response and high-efficiency control strategy of an induction motor," IEEE Trans. Ind. Appl., vol. LA-22, pp. 820-827, Set./Oct. 1986.

\section{NOMENCLATURE}

Estimated quantities.

* Commanded or reference quantities.

$e \quad$ Arbitrarily aligned synchronous frame quantity.

if, $s f \quad$ Rotor and stator flux synchronous frame quantities, respectively.

$s \quad$ Stationary frame quantity.

$f_{q d r}, f_{q d s}$ Complex rotor and stator quantities, respectively, i.e., $f_{q d r}=f_{q r}-j f_{d r}$.

$p \quad$ Differential operator; $p=j \omega_{e}$ at steady state.

$T_{e}^{\prime} \quad$ Normalized electromagnetic torque, $T_{e}=$ $3 / 2 P / 2 T_{e}^{\prime}$.

$\lambda_{r}, \lambda_{s} \quad$ Rotor and stator flux magnitudes, respectively.

$\sigma L_{s} \quad$ Stator transient inductance, $L_{s}-L_{m}^{2} / L_{r}$.

$\tau_{r} \quad$ Rotor time constant; $L_{r} / r_{r}$

$\theta_{r} \quad$ rotor position (elec. rad).

$\theta_{r f}, \theta_{s f} \quad$ Rotor and stator flux angles, respectively (elec. rad).

$\omega_{e} \quad$ Fundamental excitation frequency ( $\left.\mathrm{rad} / \mathrm{s}\right)$.

$\omega_{r}, \omega_{s} \quad$ Rotor velocity and slip frequency, respectively $(\mathrm{rad} / \mathrm{s})$. 


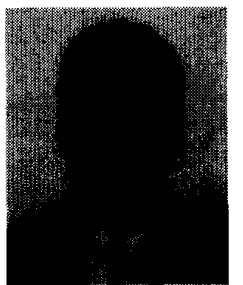

Patrick L. Jansen (S'92) received the B.S., M.S., and $\mathrm{Ph} . \mathrm{D}$. degrees in electrical engineering from the University of Wisconsin, Madison, in 1985, 1987 , and 1993, respectively.

From 1987 to 1989 he was an experimental Scientist in the Commonwealth Scientific and Industrial Research Organization (CSIRO), Division of Applied Physics, New South Wales, Australia and a Development Engineer at $\mathrm{Ma}$ chine Dynamics Pty. Ltd., Victoria, Australia. His dissertation research included the integration of electric machine design and transducerless position and velocity estimation, observer-based direct field orientation, and linear induction machine design for high-speed material transport systems. He is currently involved with the development of ac drive locomotives for the Morrison Knudsen Corporation, Boise, ID.

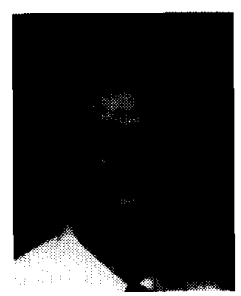

Robert D. Lorenz (SM'91) received the B.S., M.S., and Ph.D. degrees from the University of Wisconsin, Madison, in 1969, 1970, and 1984, respectively.

Since 1984 he has been a member of the Faculty of the University of Wisconsin, Madison, where he is a Professor of Mechanical Engineering and of Electrical and Computer Engineering. In this position, he acts as Associate Director of the Wisconsin Electric Machines and Power Electronics Consortium and as CoDirector of the Advanced Automation and Robotics Consorhum. He was a visiting Research Professor in the Electrical Drives Group of the Catholic University of Leuven, Leuven, Belgium and in the Electrical Drives Institute of the Technical University of Aachen, Germany, in the Summer of 1989 and the Summers of 1987 and 1991, respectively. In 1969-1970 he did his Master thesis research at the Technical University of Aachen, Germany. From 1972 to 1982 he was a member of the Research Staff at the Gleason Works, Rochester, NY. His current research interests include sensor integrated electromagnetic actuator technologies, real-time digital signal processing and estimation techniques, and ac drive and high-precision machine control technologies.

Dr. Lorenz is a Chairman of the IEEE IAS Industrial Drives Committee, and is a member of the Industrial Automation and Control Committee, the Electrical Machines Committee, and the Industrial Power Converter Committee. He is an active consultant to many organizations and is a Registered Professional Engineer in the states of New York and Wisconsin. He is a member of the ASME, the ISA, and the SPIE.

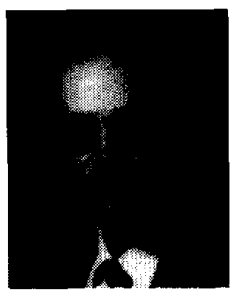

Donald W. Novotny (F'87) received the B.S. and M.S. degrees in electrical engineering from the Illinois Institute of Technology, Chicago, in 1956 and 1957, and the Ph.D. degree from the University of Wisconsin, Madison, in 1961.

Since 1961 he has been a member of the Faculty at the University of Wisconsin, Madison, where he is currently Grainger Professor of Electric Machines and Power Electronics and Co-Director of the Wisconsin Electric Machines and Power Electronics Consortium (WEMPEC). He served as Chairman of the Electrical and Computer Engineering Department from 1976 to 1980 and as an Associate Director of the University-Industry Research Program from 1972 to 1974 and from 1980 to the present. He has been active as a consultant to many organizations including Marathon Electric Company, Borg Warner Corporation, Barber Coleman Company, Otis Elevator Corporation, Allen Bradley Company, Eaton Corporation, and the Wisconsin Department of Natural Resources. He has also been a visiting Professor at Montana State University, the Technical University of Eindhoven, Eindhoven, The Netherlands, the Catholic University of Leuven, Leuven, Belgium, and a Fulbright Lecturer at the University of Ghent, Ghent, Belgium. He has published over 90 technical articles on electric machines, variable frequency drives, and power electronic control of industrial systems, several of which have received prize paper awards from the IEEE Industry Applications Society.

Dr. Novotny is a member of ASEE, Sigma Xi, Eta Kappa Nu, and Tau Beta $\mathrm{Pi}$ and is a Registered Professional Engineer in Wisconsin. 